\title{
La argumentación por medio de la comunidad de indagación
}

\author{
Sebastián Álvarez Londoño* \\ Olga Jeaneth Medina Cáceres** \\ Camilo Alejandro Grimaldos Fuentes
}

Artículo de investigación

Fecha de Recepción: 30 octubre 2017.

Fecha de Aprobación: 23 marzo 2018.

\section{Resumen}

El objetivo del presente artículo es compartir los avances del trabajo de investigación homónimo que se adelanta en la Maestría en Educación con Énfasis Profundización de la Universidad Pedagógica y Tecnológica de Colombia. Se presentará una reflexión acerca de los hallazgos teóricos sobre el desarrollo de competencias argumentativas por medio de la implementación de la comunidad de indagación, según lo propuesto en el programa de Filosofía para Niños (FpN). La metodología parte de una revisión bibliográfica, especialmente de artículos de investigación encontrados en revistas indexadas; posteriormente se elaboran fichas temáticas para hacer un análisis de las principales categorías relacionadas con el tema.

Para establecer la correlación entre las competencias argumentativas y la comunidad de indagación, el presente escrito se organiza temáticamente en tres apartados; el primero menciona los elementos fundamentales de $\mathrm{FpN}$ y comunidad de indagación; planteando cómo este es un proyecto ético-político, en segundo lugar se mostrará cómo el programa FpN contribuye al desarrollo de pensamiento crítico y ético; finalmente se hará alusión a cómo la implementación de una comunidad de indagación contribuye al desarrollo de
* Instituto Técnico Industrial Rafael Reyes - Boyacá Colombia sebasophos@gmail.com *Instituto Técnico Industrial Rafael Reyes - Boyacá Colombia olgajemedinaca@gmail.com *** Instituto Técnico Industrial Rafael Reyes - Duitama, - Boyacá Colombia

kal-el-gri@botmail.com

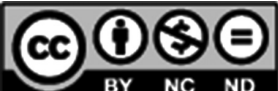


la argumentación en niños y jóvenes, Palabras clave: Filosofía para Niños, favoreciendo la formación de una argumentación, comunidad de ciudadanía democrática.

indagación, pensamiento crítico, pensamiento ético, democracia. 


\section{Introducción}

La educación en tanto derecho universal, debe ser entendida como un proceso continuo de desarrollo de competencias físicas, intelectuales y morales. En este proceso y a través de la experiencia docente, se hace evidente la necesidad de desarrollar el pensamiento crítico a través del continuo mejoramiento de las prácticas educativas; la competencia argumentativa, como partefundamental de este tipo de pensamiento, representa, en los seres humanos, una forma especial de comunicación, donde se determina lo que es válido, se apropia el conocimiento y se dirimen disensos de forma pacífica.

Ahora bien, durante el desarrollo de la actividad docente en el Instituto Técnico Industrial Rafael Reyes - Duitama, en varias ocasiones, se ha hecho evidente la forma en que, desprovistos de una justificación racional que fundamente sus convicciones y disposiciones de conducta, los estudiantes son fácil presa de la injerencia de la autoridad, la tradición o la presión de grupo. Esta actitud se hace evidente especialmente en los casos en los cuales, en medio de una indagación de preconceptos sobre un problema en filosofía política o moral, o tras buscar mediación para un conflicto entre dicentes, éstos (mayoritariamente) resultan incapaces de estructurar argumentos propios desde los cuales defender su posición.

Así mismo, el Proyecto Educativo Institucional del colegio está direccionado por unos principios que rigen la formación integral de los educandos y que encaminan la labor educativa hacia formar en la democracia, actuar con criterio, desenvolverse en la sociedad y desarrollar la capacidad de reflexión y toma de decisiones.

El propósito de esta investigación es entonces, mejorar el desarrollo de procesos argumentativos de tal forma que contribuyan a encaminar a nuestros estudiantes a una forma de pensamiento más crítica, autónoma y reflexiva, que los habilite como sujetos aptos para ser parte activa de la vida pública en el contexto de las prácticas democráticas y la participación ciudadana.

Así pues, se busca indagar por la forma en que el programa de Filosofía para niños $(\mathrm{FpN})$ contribuye a este propósito a través de su principal estrategia llamada "comunidad de indagación", la cual está encaminada a la reflexión colectiva, el pensamiento crítico, el pensamiento ético, la autonomía de criterio y la democracia.

De esta forma, el presente artículo busca demostrar que la escuela, con miras a lograr una educación que fomente la formación de ciudadanos libres y autónomos, debe superar el paradigma tradicional en el cual la enseñanza está en el centro del proceso en el aula. Es urgente que la educación parta de los propios intereses y preguntas de los niños; debemos asumir un nuevo paradigma en el que "la enseñanza cobra su auténtico sentido sólo en la medida en que genera en el niño procesos de pensamiento propios, deliberados y autocorrectivos. Se trata, pues, de enseñar para pensar, más que
El propósito de esta investigación es entonces, mejorar el desarrollo de procesos argumentativos de tal forma que contribuyan a encaminar a nuestros estudiantes a una forma de pensamiento más crítica, autónoma y reflexiva 


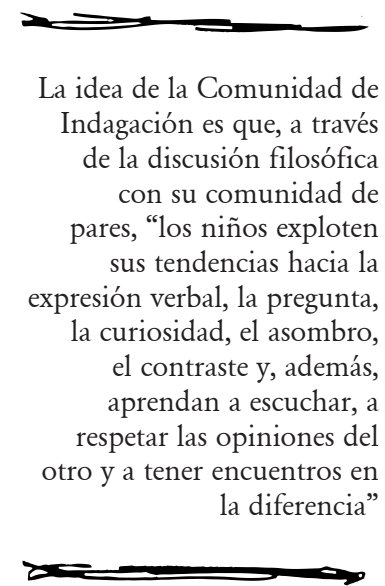

de enseñar para aprender” (Pineda, 2004, p. 9) Así, es preciso cultivar en los estudiantes la posibilidad de generar y comunicar soluciones propias a los cuestionamientos que surgen a partir de su contacto con el mundo. En este sentido, si bien es cierto que los niños a menudo son capaces de generar hipótesis que intentan saciar su capacidad de asombro; es necesario confrontarlos con las preguntas y la discusión a través de las cuales lograran superar las limitaciones que impone la lógica natural; de lo contrario, en lugar de comprender el mundo, los niños se encontrarían en un "caos de sensaciones dispersas o de pensamientos sin conexión” (Pineda, 2004, p. 7)

La idea de la Comunidad de Indagación es que, a través de la discusión filosófica con su comunidad de pares, "los niños exploten sus tendencias hacia la expresión verbal, la pregunta, la curiosidad, el asombro, el contraste y, además, aprendan a escuchar, a respetar las opiniones del otro y a tener encuentros en la diferencia" (Arias, Carreño y Mariño, 2016, p. 239). De esta forma, a través de generar en los estudiantes una actitud filosófica y un pensamiento crítico, ético y argumental; ellos tendrán "las bases necesarias para desempeñarse de manera responsable en la vida pública y privada" (Fajardo, 2016, p. 76) ya que se desarrollará la actitud crítica que les va a permitir "enfrentar su vida, valiéndose de la voluntad para no ser gobernados por otros, sino gobernarse a sí mismos" (Arias et al., 2016, p. 239) y producir procesos de transformación (Mariño, Pulido \& Morales, 2016).

\section{Filosofía para Niños y la Comunidad de Indagación}

\section{Argumentación}

La argumentación es una herramienta indispensable para el aprendizaje de los niños por cuanto es un proceso que implica la interrelación de elementos tales como la comunicación, la interacción y la reflexión comunitaria con el objetivo común de deliberar o debatir acerca de un tema que les interesa, hecho que implica la generación y producción de ideas, cuyo producto final serán conclusiones logradas a partir del consenso.

La escuela como espacio de aprendizaje generador de conocimientos, donde se facilita la interacción entre estudiantes y docentes, debe propiciar escenarios de argumentación, puesto que el desarrollo de esta capacidad se constituye en uno de los factores clave para el éxito individual y social dentro del contexto de una sociedad democrática.

El Instituto Técnico Industrial Rafael Reyes en su PEI propende por "Crear conciencia y responsabilidad en el estudiante para que aprenda a educarse, a ser persona, a actuar con criterio y desenvolverse en la sociedad verdaderamente democrática.” (p.10). Es por ello que el trabajo de investigación del que surge esta ponencia, armoniza con los intereses de la comunidad puesto que busca la formación de ciudadanos autónomos y capaces de tomar sus propias decisiones quienes podrán construir 
la democracia, pacífica, ética, vibrante y participativa que nuestro contexto tanto reclama.

El proyecto de Filosofía para niños está ampliamente difundido; prueba de ello son el gran número de artículos académicos e investigaciones que se realizan cada año en torno a la implementación de dicho proyecto y su afectación en el desarrollo del pensamiento, la comunicación $\mathrm{y}$ las emociones. Algunos de los aspectos más relevantes de los que pretenden dar cuenta las publicaciones académicas en el campo de la filosofía para niños, tienen que ver con las habilidades argumentales, la educación para la ciudadanía y el desarrollo del pensamiento filosófico en la infancia.

$\mathrm{FpN}$ es una propuesta que pretende en principio, a partir del dialogo filosófico, generar habilidades superiores del pensamiento en niños y adolescentes. Surge a finales de los años sesenta cuando el profesor universitario norteamericano Matthew Lipman se percata de la dificultad que tienen sus estudiantes a la hora de redactar textos con coherencia y sus deficiencias en el desarrollo de competencias cognitivas evidenciadas en la enorme dificultad que les implicaba realizar inferencias y analogías; así, se ve instado a construir un proyecto educativo que consiga superar esos inconvenientes. Lipman parte de considerar un error la forma en que tradicionalmente se ha dejado a la educación superior el desarrollo del pensamiento crítico y reflexivo. Así, el filósofo canadiense propone que estas habilidades deben ser desarrolladas desde la edad preescolar, de tal forma que pensar de manera crítica y reflexiva se convierta en un hábito desde la infancia (Pineda, 2004, pp. 58-59).

Los objetivos fundamentales de Filosofía para Niños, según su mayor promotor en Colombia, Diego Antonio Pineda (2004) son seis:

1. El desarrollo de la capacidad de razonamiento: se busca que los niños cultiven su capacidad de razonamiento, en todos los ámbitos, aplicando diversos tipos de lógica. Esto se consigue a través de desarrollar en los estudiantes un especial cuidado por su pensamiento y el uso del lenguaje; creando hábitos de coherencia, atención a las falacias y el continuo examen de los razonamientos.

2. Desarrollo de la comprensión ética: contribuyendo al desarrollo moral y empatía, no a través la normatividad axiológica; sino desde el proceso de investigación ética que busca identificar situaciones morales, ofrecer valoraciones adecuadas y someterlas a examen.

3. Desarrollo de la capacidad para descubrir el significado de la experiencia: que incluye tanto el significado de lo empírico como el de las experiencias que son producto de las interacciones entre símbolos (textos, obras de arte, etc.). Así, se busca que el estudiante pueda aprender a establecer diferentes tipos de relaciones: semejanza, diferencia, partetodo, medios-fines) etc.

4. Desarrollo de la creatividad: a través de la promoción de métodos alternativos de pensamiento. Este tipo de creatividad está fuertemente ligad al
FpN es una propuesta que pretende en principio, a partir del dialogo filosófico, generar habilidades superiores del pensamiento en niños y adolescentes. 
Por consiguiente, la educación sería el arte de volver este órgano del alma del modo más fácil y eficaz en que pueda ser vuelto, mas no como si se le infundiera la vista, puesto que ya la posee, sino, en caso de que sí lo haya girado incorrectamente y no mire a donde debe, posibilitando la corrección. (Platón, 1986, p. 344). trabajo lógico, especialmente cuando el dialogo filosófico versa sobre cuestiones relacionadas con el arte.

5. Crecimiento personal e interpersonal: favoreciendo la madurez emocional, la autoestima y la auto comprensión.

6. La formación en valores democráticos para la convivencia ciudadana: generando pensamiento crítico que permita construir una ciudadanía autónoma y divergente; al mismo tiempo que pacífica y tolerante. (pp. 71-74)

\section{La comunidad de Indagación}

El centro del programa de Filosofía para Niños está en la Comunidad de Indagación. La idea es que, a partir de preguntas que los propios estudiantes construyen y proponen (tomando como incentivo inicial un texto, capítulo de una novela, video, obra de arte etc.) se genere un diálogo filosófico en el aula donde son los estudiantes los protagonistas y el docente tiene el papel de acompañante.

Ahora bien, no se trata de imponer ideas, ni de buscar quién tiene la razón, sino de buscar, a partir de la interacción de la comunidad, cultivar una actitud razonable que permita entender la perspectiva de los otros y vincularla a las propias reflexiones.

Así, la comunidad de indagación y el proyecto de Filosofía para niños no parte de un conjunto de temas prefijados para ser discutidos, no hay lista de contenidos. Son los individuos que conforman la comunidad los que construyen, desde sus propios intereses la orientación que toma la indagación, propiciando de esta forma el aprendizaje desde la socialización (Pineda, 2004, p.88). Se supera entonces la transmisión pasiva de valores, es una herramienta para el desarrollo de la ciudadanía ya que promueve el desarrollo cognitivo (que favorece la reflexión individual) y las habilidades comunicativas (que favorecen la discusión, debate y confrontación de ideas).

Es la puesta en práctica de Filosofía para Niños, en tanto desarrolla competencias del pensamiento y la discusión, promoviendo en los estudiantes las virtudes dianoéticas descritas por Aristóteles en la ética a Nicómaco. Esto se da por la vía de poner al estudiante, sus ideas y su propia perspectiva del mundo, la sociedad y el yo, en el centro de la práctica pedagógica.

Por consiguiente, la educación sería el arte de volver este órgano del alma del modo más fácil y eficaz en que pueda ser vuelto, mas no como si se le infundiera la vista, puesto que ya la posee, sino, en caso de que sí lo haya girado incorrectamente y no mire a donde debe, posibilitando la corrección. (Platón, 1986, p. 344).

Es entonces posible ver cómo estructurar una propuesta desde la argumentación y comunidad de indagación, genera en los estudiantes un conjunto de virtudes de carácter ético, epistémico y emocional que por ende 
promueven algunas de las pretensiones universales que determinan una buena comunicación y por vía de esta, la democracia.

La comunidad de indagación es entonces un vehículo para generar espacios democráticos al interior de las instituciones educativas en tanto promueve el diálogo razonado, en donde la validez argumental y no la autoridad o la presión de la opinión mayoritaria, se erige como condición de acuerdo.

Una comunidad de indagación es donde se ofrecen argumentos, se examinan razones, se ponen en juego diversos puntos de vista y se examina hasta qué punto es razonable cada uno de dichos puntos de vista, etc.; en síntesis, una comunidad argumentativa. (Pineda, 2004, p. 88)

\section{El pensamiento Crítico y el Pensamiento Ético en Filosofía para Niños}

\section{El Pensamiento Crítico}

Según Pineda (2009) Podemos entender por pensamiento crítico aquel que se basa adecuadamente en criterios, es decir, es el tipo de pensamiento que es capaz de determinar cuáles son los principios adecuados que deben regir una decisión o un curso de acción determinado.

En este sentido, es claro que la comunidad de indagación, gracias a que se propone alentar a los niños y jóvenes en la tarea de justificar sus acciones y opiniones con adecuación al contexto y a los principios básicos con que la lógica; se convierten en una herramienta poderosa para propiciar el pensamiento crítico.

Por otra parte, el pensamiento crítico junto con el pensamiento creativo, generan lo que Lipman llama "pensamiento de orden superior"

Así, por un lado, tenemos un juicio crítico que fluye por el pensamiento creativo y un juicio creativo que cursa a través del pensamiento crítico. Por otro lado, tenemos un pensamiento crítico $y$ un pensamiento creativo que se penetran mutuamente generando el pensamiento de orden superior (Lipman, 1991, p. 118)

De esta forma, la comunidad de indagación, gracias a su particular forma de plantear el aprendizaje desde los intereses e inquietudes de los propios estudiantes, contribuye a desarrollar en ellos, por una parte, la creatividad necesaria para afrontar los dilemas e inquietudes que surgen del diálogo filosófico entre pares; y por otra, el pensamiento crítico que les permite tomar una postura argumentalmente justificada frente a esos mismos dilemas e inquietudes. De esta forma, FpN se convierte en un potente propiciador del desarrollo del pensamiento superior en niños, niñas y jóvenes. "Por ello para que acontezca el pensamiento crítico en las aulas es necesario que se trabaje sobre materiales curriculares de gran potencialidad como los narrativos y a partir de una pedagogía compleja desarrollada a partir de la comunidad de investigación” (Lipman, 1991, p. 118) 


\section{Pensamiento Ético}

Usualmente se relaciona a FpN con el desarrollo de habilidades cognitivas; si bien la capacidad de pensar con corrección formal es fundamental, tanto para este como de cualquier proyecto educativo que pretenda formar personas razonables, libres y autónomas; la idea de los promotores de $\mathrm{FpN}$ no se limita a este cometido. "En tal sentido, FpN no es sólo un programa para el desarrollo de habilidades cognitivas; es, sobre todo, un programa de desarrollo social. Su intención es de carácter ético-político, más que cognitivo-instrumental". (Pineda, 2004 p.49). Así, FpN es no es exclusivamente una estrategia para mejorar el desempeño cognitivo de los infantes; es, ante todo, tal cual lo propone Pineda, un proyecto éticopolítico.

Ahora bien, la capacidad para generar argumentos válidos, y diferenciarlos de aquellos que no lo son, no es ajena a la formación en el campo de la ética. $\mathrm{FpN}$ es una propuesta de formación ética, no en el sentido de inculcar a las nuevas generaciones valores de la tradición cultural, sino que "El trabajo ético en $\mathrm{FpN}$ está más preocupado por desarrollar en el estudiante la capacidad de buen juicio en cuestiones morales, lo que implica cultivar el buen razonamiento moral y la capacidad de análisis de las situaciones morales en que nos vemos implicados" (Pineda, 2004, p.69). Así, FpN se aleja de la idea dogmática de una formación ética transmisionista, y fieles al espíritu de formación de criterio desde la comunidad de indagación, se propone formar pensamiento ético que le permita a los niños aplicar criterios adecuados en sus juicios morales.

Así, la razonabilidad es parte fundamental del desarrollo del pensamiento ético, dado que "el ser razonable es precisamente uno de los elementos básicos de una vida ética, pues incluye a la vez ser capaz de discernir acertadamente entre diversas opciones en conflicto y hacerlo con base en la aplicación de criterios relevantes" (Pineda, 2009, p. 328). Así, el pensamiento ético alude a la habilidad para formar criterios adecuados que rijan las acciones desde el ámbito moral.

Un aspecto fundamental del desarrollo del pensamiento ético a partir de la comunidad de indagación tiene que ver con la habilidad para, desde la empatía y la tolerancia, entrar en diálogo con opiniones divergentes:

De la misma forma que se vislumbran pensamientos críticos, lo hacen los pensamientos éticos. Interactuar con diversas formas de pensar, sentir, interpretar, proyectar, hablar, preguntar, imaginar y crear, genera situaciones de desacuerdo, por lo que se hace necesario discutir la circunstancia en sí misma, escuchar y reflexionar sobre las diferentes opiniones y aceptar sugerencias, de modo que se llegue a un acuerdo sin pretender nunca un pensamiento homogéneo. (Arias, Carreño y Mariño, 2016, p. 256)

La comunidad de indagación es entonces, a través del desarrollo del 
pensamiento crítico, una estrategia para desarrollar el pensamiento ético, pues al mismo tiempo en que el diálogo filosófico entre pares contribuye a poner en práctica la capacidad crítica y creativa, lo hace también con la empatía, la tolerancia, el respeto y la capacidad para usar criterios que permiten que las acciones de los niños, niñas y jóvenes, se adecuen a los principios éticos que determinan una sociedad democrática.

\section{La Argumentación en la Comunidad de Indagación}

\section{La filosofía como promotora del saber racional}

La prohibición de asombrarse y preguntarse se transmite de generación en generación. En poco tiempo, los niños que ahora están en la escuela llegarán a ser padres. Si de algún modo podemos preservar su natural sentido de asombro, su apertura a la búsqueda de significados, su anhelo de comprensión del por qué de las cosas, puede haber una esperanza de que esa generación no sirva a sus propios hijos como modelo de aceptación acrítica. (Lipman, Sharp y Oscanyan, 1998, p. 89)

En la era de la posverdad ${ }^{1}$ en que parecemos perder nuestra capacidad para discernir entre una inferencia válida y una falacia, en la que no logramos diferenciar una buena razón de aquellas que no lo son y en la que se exige cada vez menos que nuestras acciones estén fundamentadas desde la razón; la escuela debe fungir como promotora para la racionalidad humana.
La filosofía, como conjunto de saberes que intentan establecer, de manera lógico-racional, los principios fundamentales del conocimiento del mundo y del obrar del ser humano en tanto individuo y en su función socio-comunitaria, es el marco de referencia a partir del cual, todos los demás ámbitos del conocimiento, la comunicación y la praxis humana, se encuentran determinados. "De este modo, la tarea del filósofo consiste en examinar, validar o invalidar los conceptos, en crear los propios y, en innovar y establecer un sistema para analizar su tiempo y su cultura" (Arias, Carreño y Mariño, 2016, p. 239). Así, a lo largo de la historia, la filosofía se ha encargado de desarrollar las habilidades del pensamiento que permiten a sus practicantes cuestionar, indagar, inferir y discernir a partir de los más variados conceptos. Es por esto que se hace necesario, desde el ámbito escolar, promover el ejercicio filosófico y las herramientas que posibilitan su desarrollo como propiciador de la racionalidad que permite una vida realmente libre.

Es necesario que el quehacer filosófico deje de estar relegado a la condición de actividad reservada a unos pocos iniciados que solo ellos entienden; nuestra realidad cotidiana hace urgente que la actividad filosófica tenga presencia en las aulas como herramienta esencial a la hora de formar estudiantes con la capacidad de razonar de forma efectiva en los diversos contextos que el mundo y su futura condición de ciudadanos les presentan:
1 El Diccionario de Oxford define que posverdad "denota circunstancias en que los hechos objetivos influyen menos en la formación de la opinión pública, que los llamamientos a la emoción y a la creencia personal". 


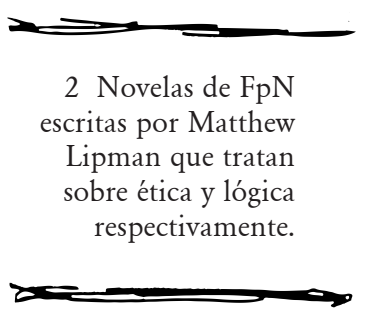

La construcción de argumentos correctos es una de las tareas esenciales del trabajo filosófico, y los niños están en capacidad de identificar las reglas generales de un buen razonamiento y de aplicarlas en situaciones cotidianas, tal como lo hacen los niños que participan de una discusión argumentativa como las que aparecen en textos como Elisa o El descubrimiento de Harry $^{2}$. (Pineda, 2004, p.10)

\section{La comunidad de indagación como estrategia para promover la argumentación}

Durante la primera infancia, los niños adquieren, junto con el lenguaje, las reglas iniciales de la lógica a partir de la interacción con su núcleo familiar "Los usos familiares que les son insinuados a los niños, así como las interacciones que ellos tienen con su ambiente, motivan la escogencia que hacen por inferencias válidas en vez de inválidas" (Lipman, s.f., p. 4). A partir de ahí, el desarrollo de los procesos de pensamiento debe tener el apoyo decidido de la escuela; tal como propone Diego Pineda "la enseñanza cobra su auténtico sentido sólo en la medida en que genera en el niño procesos de pensamiento propios, deliberados y autocorrectivos. Se trata, pues, de enseñar para pensar, más que de enseñar para aprender" (Pineda, 2004, p. 9) Así, es preciso cultivar en los estudiantes la posibilidad de generar y comunicar soluciones propias a los cuestionamientos que surgen a partir de su contacto con el mundo. En este sentido, si bien es cierto que los niños a menudo son capaces de generar hipótesis que intentan saciar su capacidad de asombro; es necesario confrontarlos con las preguntas y la discusión a través de las cuales lograran superar las limitaciones que impone la lógica natural; de lo contrario, en lugar de comprender el mundo, los niños se encontrarían en un "caos de sensaciones dispersas o de pensamientos sin conexión" (Pineda, 2004, p. 7)

Ahora bien, el desarrollo de esta habilidad para argumentar de forma racional no se desarrolla introduciendo la filosofía en la escuela como una mera evocación de los hitos en la historia del pensamiento occidental ni desarrollado ejercicios de lógica formal: "Es obvio que tal desarrollo no se da con la simple explicación de los principios de la lógica o forzando a los alumnos a que se comprometan en la solución de exhaustivos ejercicios de lógica; y mucho menos dándoles ejemplos de cuán maravillosamente razonamos nosotros, los adultos." (Lipman, s.f., pp. 2-3)

Es por esto que en el programa de Filosofía para Niños se propone la comunidad de indagación como base del desarrollo de competencias racionales, permitiendo a niños $\mathrm{y}$ adolescentes a depurar su habilidad para estructurar argumentos válidos e identificar buenas razones a través de la puesta en práctica del diálogo filosófico:

En el salón de clase los alumnos discuten esos descubrimientos de manera cooperativa. Si algunos realizan generalizaciones, otros 
pueden ofrecer contraejemplos; si algunos emiten opiniones sin razones, éstas les son prontamente exigidas. Ellos, poco a poco, van descubriendo inconsistencias en sus propios pensamientos. (Lipman, s.f., p.9)

El pensar sobre el propio pensar y la construcción de argumentos correctos, como tarea esencial del trabajo filosófico, está también en el centro de FpN. Así, según lo señalan Catalani y Velasco (2009), dentro de la propuesta de Matthew Lipman, creador de FpN, podemos encontrar tres enfoques desde los que se aborda la lógica: Lógica Formal, que incluye cuestionamientos que tradicionalmente hacen parte de la llamada lógica formal, pero también de la lógica informal; lógica de las buenas razones y la lógica del actuar racional.

La lógica formal abarca la lógica aristotélica y la lógica de relaciones; además incluye el silogismo hipotético, la relación entre parte y todo, el esquema de las cuatro posibilidades, la inducción y las tautologías (Catalani y Velasco, 2009, p. 289). Así, la lógica formal incluye tanto los modelos abstractos de argumentos que son susceptibles de ser generalizados (lógica formal) y los argumentos que, remitiendo a un contexto particular, permiten un análisis práctico que remite al lenguaje natural (lógica informal).

La lógica de las buenas razones, por el contrario, no se enfoca en dar reglas específicas para la construcción de argumentos, sino que prioriza el buscar y evaluar razones en una situación determinada (Catalani y Velasco, 2009, p. 308). Así, se parte de la idea de que una persona razonable es aquella que es capaz de diferenciar una buena razón de aquella que no lo es, tanto en su propio discurso como en el de sus interlocutores (Pineda, 2009, p. 318); en este sentido: "Una buena razón implica una argumentación cuidadosa, pero también una sensibilidad fuerte hacia las necesidades del contexto, así como el ejercicio permanente de la autocorrección” (Pineda, 2004, p. 10).

Así, discernir cuando una razón es una "buena razón" depende de usar el criterio adecuado de acuerdo al contexto en el cual se está llevando a cabo la argumentación, pues no es lo mismo, por ejemplo, una buena razón para defender una hipótesis científica (que debe estar basada en hechos objetivamente observables) que una buena razón que me permita tomar postura en torno a un dilema moral: Una razón aceptable en cuestiones morales es, entonces, aquella que justifica (esto es, que "hace justa") una acción, es decir, que muestra que dicha acción sería deseable y que debería ser realizada (Pineda, 2009, p. 318). Queda claro entonces, que el dialogo filosófico propio de la comunidad de indagación promueve en los estudiantes la capacidad para brindar, exigir y evaluar argumentos en adecuación al contexto.

La lógica del actuar racional por su parte, pone énfasis en la práctica cotidiana del pensamiento reflexivo con lo cual tiene por objetivo "el uso del pensamiento reflexivo para la obtención de comportamiento razonable” (Catalani y Velazco, 2009, 
p. 312). Así, el programa de filosofía para niños a través de la comunidad de indagación no solamente contribuye a la interiorización de las reglas formales que permiten la identificación de inferencias válidas, sino que también posibilita que se desarrolle la capacidad de comprender los argumentos en su propio contexto y se actúe en consecuencia. Es en este sentido en el que el diálogo filosófico, a través del desarrollo de la competencia argumentativa deviene en formador de ciudadanos.

\section{La comunidad de indagación, la argumentación y la formación ciudadana}

El Ministerio de Educación Nacional (2004), propone un conjunto de competencias cognitivas que buscan generar en los estudiantes la capacidad de desarrollar diversos procesos mentales que les permitan un eficaz ejercicio de la ciudadanía. De la misma forma, se proponen competencias comunicativas que buscan generar la capacidad de establecer diálogos efectivos en las diversas posibilidades de la interacción social. Además, propone un conjunto de competencias emocionales que apuntan hacia la empatía y la capacidad resolver pacíficamente los conflictos.

Ahora bien, la competencia argumental es indispensable para la construcción de la ciudadanía y la democracia en tanto ésta presupone la participación de sujetos libres, capaces de tener una perspectiva racional propia y entablar con las demás discusiones que, en lugar de convertirse en una estrategia retórica para alcanzar los propios fines, busquen un consenso sujeto a la postulación de argumentos válidos. Tal como lo propone Nussbaum (2010): "Cuando se pierden de vista los argumentos, las personas se dejan llevar con facilidad por la fama o el prestigio del orador, o por el consenso de la cultura de pares" (p. 79)

Por otra parte, sostiene también Nussbaum (2010) "La idea de ser responsable por los argumentos propios y de intercambiar opiniones con los demás en un entorno de respeto mutuo por la razón es fundamental para la resolución pacífica de las diferencias" (p. 83). Así pues, las personas irreflexivas son más susceptibles de ser instigadas por la autoridad, por sus pares o por sus propios prejuicios, lo cual genera un grave peligro para la defensa de la libertad individual y los derechos humanos en una sociedad cada vez más diversa y plural, en donde se hace imperativo el respeto por la diferencia y, al mismo tiempo, la búsqueda racional de consensos sobre los principios ético-políticos que deben regir la vida comunitaria.

Así entonces, podemos señalar que la comunidad de indagación, como modelo de enseñanza a partir del diálogo y el debate filosófico, promueve las habilidades argumentales, comunicativas y emocionales que propician formación de ciudadanos racionales, autónomos y capaces de construir una sociedad dialogante y diversa. 
En resumen, la tarea de la lógica en $\mathrm{FpN}$ consiste en desarrollar la atención sobre cada uno de los rasgos del pensamiento. Con esto, aceptamos que las personas podemos equivocarnos al proporcionar razones, pero no que sea imposible proporcionar buenas razones. Así, la lógica se encarga del desarrollo de un estado de conciencia, por ello, las estrategias que FpN usa no se limitan a lo cognoscitivo reúne elementos como lo estético y lo político. La lógica es una apuesta por atender a cada uno de los usos del lenguaje y la vital relación con las formas de vida como lo afirma el filósofo vienés Wittgenstein en las investigaciones filosóficas. (Schumann, 2007, p. 73)

Queda claro pues que FpN y la comunidad de indagación en tanto "diálogo entre iguales que permite la construcción de seres democráticos, políticos y éticos" (Arias et al., 2016, p. 239) promueven el trabajo comunitario, la empatía, la resolución pacífica, racional y autónoma de los conflictos y por ende la formación ciudadana.

\section{Conclusiones}

Filosofía Para Niños y la Comunidad de Indagación, como instancia fundamental que surge este proyecto educativo, lejos de lo que su nombre podría insinuar, no es una estrategia para "enseñar" en sentido tradicional los contenidos propios de la historia filosófica de occidente. Muy por el contrario, es un proyecto Éticopolítico, que pretende contribuir a la formación de ciudadanos autónomos, críticos y responsables éticamente de sus opiniones y decisiones.

La comunidad de indagación, por vía de propiciar el diálogo filosófico entre pares es un potente propiciador del desarrollo del pensamiento crítico que permite a sus integrantes adecuar sus juicios y acciones a criterios que atiendan a la razón argumental y al contexto. Esto, sumado a la necesidad de encontrar soluciones razonables a los dilemas y la confrontación de ideas, es decir, al desarrollo del pensamiento creativo, redunda en la formación del pensamiento superior.

De la misma forma, los miembros de la comunidad de indagación, en tanto preparados para usar criterios razonables y con adecuación al contexto, y, además, al estar acostumbrados a tener en cuenta en sus propios razonamientos, los razonamientos ajenos y la necesidad de justificar sus posturas; desarrollan la empatía, el respeto y la responsabilidad que conforman el pensamiento ético.

La habilidad para construir argumentos válidos y adecuados al contexto que guíen las opiniones y las disposiciones de conducta, son necesarios para el desarrollo del pensamiento crítico y del pensamiento ético. Al mismo tiempo, la habilidad argumental está en el centro de todo diálogo filosófico y, por ende, de la comunidad de indagación y de Filosofía para Niños. Así, resulta mucho más eficaz a la hora de desarrollar habilidades argumentales en los niños, proponerles un diálogo filosófico que surja de sus 
propios intereses e inquietudes que simplemente obligarlos a desarrollar ejercicios de lógica formal o mostrarles ejemplos de argumentación filosófica.

En última instancia, teniendo en cuenta que el propósito de la democracia es que el poder de tomar decisiones que afectan la vida comunitaria sea sustentado por los ciudadanos, es necesario que dichos ciudadanos estén habilitados para construir un diálogo argumental con empatía, respeto y tolerancia. Así, filosofía para niños contribuye a fundamentar las condiciones que hacen posible pasar de una democracia formal (fundamentada exclusivamente en la estructura jurídica e institucional) a una verdadera democracia, plural y participativa

\section{Referencias Bibliográficas}

Arias, C. J., Carreño, G. A. y Mariño L. A. (2016). Actitud filosófica como herramienta para pensar. Revista Universitas Philosophica, 33(66), 237-262.

Catalani, C., Velasco P. N. (2009). Sobre a lógica no programa filosofia para crianças. Revista Universitas Philosophica, 5(10), 283-316.

Fajardo, E. (2016). Hacia la caracterización de los valores democráticos y ciudadanos de las niñas y niños escolares: una mirada desde la filosofía para niños. Revista Amauta, 14(27), 71-86.

Lipman, M., Sharp, A. M. y Oscayan F. (1998). La filosofía en el aula. Madrid: Ediciones de la Torre

Lipman, M. (1991), Pensamiento complejo y educación. Madrid: Ediciones de la Torre.

Lipman, M. (s.f). La filosofía y el desarrollo del razonamiento. (Trad. Pineda, D. A.). (Documento Inédito). Pontificia Universidad Javeriana. Bogotá. Texto Original en portugués: www.bibvirt.futuro.usp.br/textos/humanas/filosofia/ pensar/pensar1-2.html

Mariño, L., Pulido, O., \& Morales, L. (2016). Actitud filosófica, infancia y formación de maestros. Praxis \& Saber, 7(15), 81-101. https://doi. org/10.19053/22160159.v7.n15.2016.5724

Ministerio de Educación Nacional. (2004). Formar para la ciudadanía ¡SÍ es posible! Recuperado de http://www.mineducacion.gov.co/1759/w3-article-75768. html

Nussbaum, M. C. (2010). Sin fines de Lucro: por qué la democracia necesita de las bumanidades. Bogotá: Panamericana. 
Pineda, D. A. (2009). On the notion of good reasons in philosophy for children. [En torno a la noción de buenas razones en Filosofía para niños] Childhood \& Philosophy, 5(10), pp. 317-338.

Pineda, D. A. (2004). Filosofía para niños: el ABC. Bogotá: Editora Beta.

Platón. (1986). República. Madrid: Editorial Gredos.

Schumann, J. A. (2007). Aportes de una teoría de la argumentación al diálogo filosófico. En Filosofía para Niños: Ideas Fundamentales y Perspectivas Sociales. (pp. 69-86). Bogotá: Corporación Universitaria Minuto de Dios. 